\title{
O transtorno bipolar: senso comum $x$ a visão psicopatológica
}

\author{
Bipolar disorder: common sense $\mathbf{x}$ the psychopathological vision \\ Trastorno bipolar: sentido común x la visión psicopatológica
}

Recebido: 14/09/2021 | Revisado: 24/09/2021 | Aceito: 28/09/2021 | Publicado: 30/09/2021

\author{
Marcileia dos Santos Freitas \\ ORCID: https://orcid.org/0000-0002-4744-1319 \\ Centro Universitário FAMETRO, Brasil \\ E-mail: marcifreitas366@gmail.com \\ Simara de Souza Mendes \\ ORCID: https://orcid.org/0000-0001-8816-6432 \\ Centro Universitário FAMETRO, Brasil \\ E-mail: simarespsi@gmail.com \\ Julio Cesar Pinto de Souza \\ ORCID: https://orcid.org/0000-0003-3622-1393 \\ Centro Universitário FAMETRO, Brasil \\ E-mail: cmte01@yahoo.com.br
}

\begin{abstract}
Resumo
O Transtorno de Humor Bipolar (THB) se caracteriza por mudança de humor, podendo ser eufórica ou depressiva, sua intensidade e sintomas variam de pessoa para pessoa. Este transtorno afeta igualmente homens e mulheres. Existem muitos tabus e preconceito com pessoas que tem o THB o que dificulta, em muitos casos, a ida dos pacientes aos médicos para exames e diagnóstico. Esta pesquisa teve como objetivo discutir sobre o THB a partir das perspectivas do senso comum e da psicopatologia, utilizando-se de uma pesquisa de procedimento bibliográfico e abordagem qualitativa. O levantamento dos dados foi realizado nas plataformas científicas Scielo e Capes. O estudo mostrou que, pelo senso comum, o portador do THB pode trazer grandes riscos à sociedade, que o vê como um doente mental sem controle ou cura, sendo necessário afastar essas pessoas do convívio social. O tratamento não é muito divulgado, causando uma perpetuação do preconceito em relação ao THB. As poucas informações sobre o transtorno e as atitudes preconceituosas, levam muitos pacientes a sofrer com a marginalização e durante o episódio depressivo, em alguns casos, a tentativa de suicídio. O grande desafio dos profissionais da saúde mental é levar conhecimento do transtorno à sociedade, de modo a reduzir esse estigma acerca do THB.
\end{abstract}

Palavras-chave: Transtorno de humor bipolar; Senso comum; Preconceito; Suicídio.

\begin{abstract}
Bipolar Mood Disorder (THB) is characterized by mood change, which can be euphoric or depressive, its intensity and symptoms vary from person to person. This disorder also affects both men and women. There are many taboos and prejudice with people who have THB which makes it difficult, in many cases, to go from patients to doctors for examinations and diagnosis. This research aimed to discuss about THB from the perspectives of common sense and psychopathology, using a bibliographic procedure research and qualitative approach. Data were collected on the Scielo and Capes scientific platforms. The study showed that, by common sense, the patient of THB can bring great risks to society, which sees him as a mental patient without control or cure, and it is necessary to keep these people from social life. The treatment is not widely publicized, causing a perpetuation of prejudice in relation to THB. The little information about the disorder and prejudiced attitudes, leads many patients to suffer from marginalization and during the depressive episode, in some cases, the suicide attempt. The great challenge of mental health professionals is to bring knowledge of the disorder to society, in order to reduce this stigma about THB.
\end{abstract}

Keywords: Bipolar mood disorder; Common sense; Prejudice; Suicide.

\begin{abstract}
o
El trastorno bipolar del estado de ánimo (THB) se caracteriza por un cambio de humor, que puede ser eufórico o depresivo, su intensidad y síntomas varían de persona a persona. Este trastorno también afecta tanto a hombres como a mujeres. Hay muchos tabúes y prejuicios con las personas que tienen THB, lo que hace que sea difícil, en muchos casos, pasar de los pacientes a los médicos para los exámenes y el diagnóstico. Esta investigación tuvo como objetivo discutir sobre la THB desde las perspectivas del sentido común y la psicopatología, utilizando un procedimiento bibliográfico de investigación y enfoque cualitativo. Los datos fueron recolectados en las plataformas científicas Scielo y Capes. El estudio demostró que, por sentido común, el paciente de THB puede traer grandes riesgos a la sociedad, que lo ve como un paciente mental sin control ni cura, y es necesario mantener a estas personas alejadas de la vida social. El tratamiento no es ampliamente publicitado, lo que provoca una perpetuación de los prejuicios en
\end{abstract}


relación con la trata de personas. La poca información sobre el trastorno y las actitudes prejuiciosas, lleva a muchos pacientes a sufrir marginación y durante el episodio depresivo, en algunos casos, el intento de suicidio. El gran reto de los profesionales de la salud mental es acercar el conocimiento del trastorno a la sociedad, con el fin de reducir este estigma sobre la trata de personas.

Keywords: Trastorno bipolar del estado de ánimo; Sentido común; Prejuicio; Suicidio.

\section{Introdução}

O mau-humor e as oscilações de humor são reações normais na vida cotidiana e, muitas vezes, pode-se identificar os eventos que estão causando esses transtornos (De Queiroz et al., 2017). Entretanto, Barlow (2015) alerta que, ao experimentarmos oscilações de humor ou estados de ânimo extremos que surgem de forma totalmente inesperada e dificultam nosso funcionamento adequado, pode-se estar diante de um Transtorno de Humor.

Estudos de Moreno et al. (2015) indicam que o Transtorno de Humor Bipolar (THB) (anteriormente conhecido como psicose maníaco-depressiva) é um transtorno do humor que se caracteriza por períodos distintos de humor extremamente alto (mania) e humor extremamente baixo (depressão), bem como episódios de humor basal do indivíduo, ou chamados, períodos de estabilidade.

Da Costa et al. (2016) comentam que o THB geralmente começa durante a adolescência e o início da idade adulta, não sendo comum o surgimento em crianças que não têm fatores de risco familiares. É raro o aparecimento desse transtorno em pessoas com mais de 60 anos, exceto quando está associado a outra psicopatologia. Uma característica desse transtorno psiquiátrico é que não existe uma prevalência em relação ao gênero, afetando igualmente homens e mulheres. Para Deminco (2019), o THB do tipo grave pode afetar 1 a $2 \%$ da população, e três vezes mais em suas formas menos graves da psicopatologia.

A bipolaridade é um transtorno mental da psique, que acontece no ser humano por diversos motivos, tendo consigo alterações, que podem variar de leves a severas no comportamento do indivíduo, acarretando oscilações extremas de humor. Tais alterações podem ser apresentadas em momentos em que o indivíduo desenvolve atitudes desequilibradas como, irritações e depressão (Silva, et al., 2020).

Segundo a Organização Mundial da Saúde (OMS), o Transtorno Bipolar está entre as dez psicopatologias psicossociais mais incapacitantes no mundo e o diagnóstico costuma ser tardio ( 8 a 10 anos de evolução), o que pode acarretar o agravamento do transtorno e maior dificuldade no tratamento e sua evolução (Dalgalarrondo, 2018)

No senso comum, a bipolaridade é vista como um eixo do humor individual, em que cada um desliza ao longo do dia, fazendo variar o seu humor e daí seus comportamentos. O estereótipo de uma pessoa com THB é de alguém criminoso que pode trazer grandes riscos para a sociedade. Essa visão equivocada de parte da sociedade quanto ao transtorno se estende também para a família que, por vezes, sofre com certa discriminação e preconceito social (De Oliveira et al., 2019).

A família das pessoas com THB "experimentam sentimentos como o medo, o desconforto e, por vezes, optam pelo afastamento social por não se sentirem seguros e por não saberem como será sua recepção" (Fassarella et al., 2019, p. 9).

O presente artigo tem como objetivo geral discutir sobre o THB a partir da perspectiva do senso comum e os reflexos para o paciente. Para conduzir essa pesquisa, foi, inicialmente, discutido o conceito de THB e, em seguida, discute-se a percepção da sociedade em relação às pessoas com THB. Por fim apresentam-se as consequências dessa marginalização.

A relevância deste estudo para a sociedade encontra-se nas reflexões sobre o preconceito acerca da psicopatologia, desmistificando questões que levam o paciente com THB a ser visto como um louco, descontrolado e perigoso. Espera-se que, com a desconstrução dessa ideia, a sociedade se sensibilizará, levando muitos a uma mudança de atitude e procedimentos.

Este trabalho também apresenta contribuições na apresentação de informações que oferecerão um melhor entendimento do transtorno por uma perspectiva psicossocial. Levar ao acadêmico o estudo e reflexão da patologia a partir de 
um contexto social contribuirá para a melhor atuação de um futuro profissional da saúde mental, que poderá atuar em comunidades e regiões longínquas onde o desconhecimento sobre a patologia leva a uma discriminação e afastamento do paciente que, por vezes, encontra um refúgio, muito vezes, na utilização de entorpecentes ou medicamentos.

Ao discorrer sobre essa visão errônea que a sociedade tem sobre o assunto, contribuiremos para a construção de uma sociedade mais tolerante e receptiva àquilo que desconhece, sendo menos preconceituosa e discriminatória. Desta maneira, a ênfase deste estudo está em trazer a sensibilização de que a bipolaridade é um transtorno que precisa ser tratada de maneira adequada, pois pode acometer qualquer indivíduo independente de gênero, cor, raça ou classe social.

\section{Percurso Metodológico}

Trata-se de uma pesquisa de procedimento bibliográfico e abordagem qualitativa. O procedimento bibliográfico é o mais utilizado quando o pesquisador busca uma percepção mais ampla e variada (holística) sobre determinado tema a partir de material já publicado sobre o assunto (Aragão, 2017), nesse caso o THB. Bell (2016) afirma que, ao utilizar-se a pesquisa bibliográfica, o pesquisador pode escolher entre as categorias de abordagens para utilizá-la, no caso desta pesquisa optou-se pela pesquisa qualitativa, visto permitindo uma reflexão sobre o material coletado (Bauer \& Gaskell, 2017).

O levantamento de dados foi realizado nas plataformas científicas Scielo, Capes e a biblioteca da faculdade Metropolitana de Manaus, no mês de junho de 2021. Para levantamento das publicações estabeleceram-se como descritores: THB, preconceito, sociedade, social, senso comum, discriminação. Como critérios de inclusão foram estabelecidos: a. Somente as publicações nacionais e no idioma português; e b. As publicações no período de 2014 a 2021.

As fontes coletadas foram analisadas em três etapas. Na primeira etapa as publicações foram comparadas e eliminadas aquelas com títulos iguais e incompletas. A segunda etapa foi realizada leitura dos resumos dos artigos e trabalhos acadêmicos e das sinopses dos livros de modo a estabelecer aqueles trabalhos que possuíam relação direta como a objeto da pesquisa. Após a eliminação do material não compatível com a proposta da pesquisa, as publicações foram submetidas a uma leitura interpretativa e reflexiva, na qual os pesquisadores buscaram levantar as ideias dos autores, suas interpretações sobre o assunto e opiniões. Ao término da análise obteve-se 9 artigos, 8 capítulos de livros e 14 trabalhos publicados em revistas e jornais.

\section{Resultados e Discussão}

\subsection{Transtorno de Humor Bipolar: considerações básicas}

A bipolaridade é um transtorno psiquiátrico que se caracteriza por mudanças de humor, podendo ser eufórica ou depressiva. A intensidade e características desse transtorno mudam de pessoa para pessoa. Alguns têm o THB com predominância de episódios maníacos e outros com predominância de episódios depressivos. Há casos em que o indivíduo apresenta episódios depressivos e maníacos com períodos e intensidade similares e períodos de estabilidade (Da Costa, 2016).

O transtorno bipolar pode ser causado por fator biológico hereditário, genético, personalidade ou eventos traumáticos, ainda não se tem conhecimento de como exatamente se adquire a psicopatologia, somente se tem conhecimento, por meio da ciência, existem vários fatores envolvidos no humor do indivíduo que provocam a psicopatologia (Petkevivius et al, 2020). Nesse viés, Scussel et al. (2016) afirmam que uma pessoa pode se tornar bipolar por acontecimentos traumáticos no decorrer de sua vida, A mudança de hábito radical do ambiente de convivência, pode levá-la a desenvolver este transtorno. No fator genético, o indivíduo pode estar mais suscetível a ter esse transtorno por ser hereditário, quando já tem no seu histórico familiar a psicopatologia.

Entre os sintomas listados por Kapczinski e Quevedo (2016) e Lima e Neto (2019) estão: humor depressivo, desinteresse, perda ou ganho excessivo de peso, sono excessivo ou insônia, agitação ou lentidão psicomotora, esgotamento 
físico ou perda de energia imediata, sensação ou sentimento de culpa, habilidade diminuída para pensar e se concentrar, pensamentos contínuos sobre morte, pensamentos suicidas.

A respeito do portador de THB, Fassarella (2019) comenta que "a pessoa 'perde' o controle de si mesma, já que em uma hora ela se apresenta com certas atitudes, e, em outra, muda drasticamente suas ações". Assim, o comportamento do indivíduo muda de uma forma incontrolável, fazendo com que esse venham sair fora da sua realidade, e ter sua própria realidade.

Por isso, o portador de TBP precisa passar por todos os tratamentos que o levará a ter um equilíbrio sobre as crises. Nesse sentido, Moreno et al. (2015) afirmam que um indivíduo com esse transtorno, quando não medicado, pode passar por diversas oscilações de humor, apresentando episódios maníacos.

Estudos de Casanova et al. (2018) relacionam os episódios maníacos são geralmente citados por pacientes como fatores que causam as explosões (como muitos chamam o quadro de irritabilidade excessiva, que podem terminar em violência e/ou transtornos sociais), caracterizados pelos seguintes aspectos: energia excepcional, inquietação, dificuldade de concentração, sentimentos de euforia (extrema felicidade), comportamento de risco, dormir mal e uso de substâncias químicas. Esses fatores podem prejudicar diretamente a pessoa em seu ambiente de trabalho, bem como o seu convívio em sociedade, como um todo.

Durante esse episódio o paciente apresenta sintomas com uma duração mínima de uma semana, o indivíduo também apresenta uma autoestima inflada, necessidade de sono reduzida, acentuada ansiedade e pensamento acelerado levando a fuga de ideias, atenção desviada e principalmente uma grave alteração no humor. Nessa fase o indivíduo sente-se eufórico e emprega toda sua energia em atividades diárias, excedendo seus limites biológicos e psicológicos (Santos, 2019).

Porém, as pessoas portadoras de THB podem apresentar episódio Hipomaníaco, no quais os sintomas são bem parecidos com o episódio maníaco, o que diferencia são dois fatores, o primeiro é a duração inicial dos sintomas que nesta fase são apresentados por quatro dias consecutivos, o segundo fator é a gravidade dos sintomas, pois nesta fase não é suficientemente grave para ocorrer prejuízo em sua vida e até mesmo hospitalização (Moreno et al., 2015).

Além do mais, Magalhães (2021) aponta que, no decorrer das crises, as mudanças de comportamento específicas para quem sofre de THB podem deixar os indivíduos em situações delicadas pois, não conseguem realizar decisões corretas se colando em situação de promiscuidade sexual com parceiros diferentes e risco de psicopatologias de transmissão sexual e separações românticas subsequentes, violação da lei, insônia e até mesmo ao suicídio.

A autoestima é outra característica nesta fase que sofre alterações, tornando-se exageradamente inflada, desta forma o indivíduo se comporta como se fosse inabalável, no que se refere ao ambiente de trabalho acredita ser o único capaz de resolver os problemas de forma geral. Outro sintoma prevalente nesta fase é a hiperatividade, o indivíduo investe suas energias em várias atividades prazerosas, destinando assim mais tempo do que o habitua (Schiavo e Nunes, 2019).

Segundo Manual Diagnóstico e Estatístico de Transtornos Mentais 5 (DSM) (2014), para ser diagnosticado com transtorno bipolar, a pessoa deve ter experimentado pelo menos um episódio de mania ou hipomania. Os profissionais de saúde mental usam o DSM-5 para diagnosticar o "tipo" de transtorno bipolar que uma pessoa pode estar enfrentando. Para determinar que categoria de transtorno bipolar uma pessoa tem, os profissionais de saúde mental avaliam o padrão dos sintomas e quão debilitada a pessoa está durante seus episódios mais graves.

\subsection{Transtorno de Humor Bipolar: tratamento e a importância da família}

O THB não tem cura, mas pode ser satisfatoriamente controlado por meio de medicamentos, os quais serão prescritos pelo médico psiquiatra. Somente esse profissional pode prescrever ou mudar a medicação. Os fármacos mais utilizados para o manejo da sintomatologia são o lítio, os anticonvulsivantes, os antipsicóticos atípicos e os antidepressivos. (Whitbourne \& 
Halgin, 2015). "O uso da medicação de forma correta e respeitando a prescrição e orientações do médico é importante para a eficácia do tratamento, entretanto, estima-se que 18 a 50\% dos pacientes apresentam falhas no cumprimento terapêutico durante algum período da psicopatologia" (De Oliveira et al., 2019, p. 158).

Kapczinski et al. (2016) e Marques et al. (2018) relatam que o tratamento medicamentoso pode ser utilizado de modo a contribuir para a qualidade de vida do paciente. Entretanto, mesmo seguindo a medicação corretamente pode-se ocorrer recaídas chegando até as internações, tendo variações nos sintomas de episódios de humor. Por isso, a utilização de alternativas de tratamento, como práticas esportivas e um bom relacionamento familiar, contribuem para a melhoria desses pacientes, podendo proporcionar melhores condições de vida.

Fassarella (2019) corrobora o pensamento dos autores acima, pois afirma que o tratamento se baseia a partir de medicações específicas e terapia, fazendo-se presente pelo decorrer de toda a vida ou por tempo indefinido, indispensável a participação dos familiares nos meios ligados ao paciente. São também indispensáveis: a participação em grupos de apoio, terapia cognitivo-comportamental, psicoeducação, terapia familiar e psicoterapia.

Então, o grupo familiar pode se tornar um grande sustentáculo para o paciente com THB, oferecendo o apoio e o acolhimento necessários para estimular o paciente a se manter no tratamento. Almeida et al. (2018) comentam a importância da família em controlar e acompanhar as mudanças de humor, preocupando-se em não menosprezar essas mudanças como sendo algo rotineiro. Ao perceber os primeiros sintomas, a família deve buscar ajuda médica, pois cabe somente ao profissional indicar o tratamento adequado a esse paciente.

A partir da discussão, verifica-se que o THB possui uma série de sintomas indesejáveis, mas o uso de medicamentos adequados ao paciente pode levar a remissão dos sintomas, oferecendo um reequilíbrio emocional. Entretanto, o THB tem um reflexo social, visto que a mudança de humor e os sintomas apresentados pelos pacientes levam muitas pessoas a se distanciarem por medo ou preconceito, fazendo o paciente se sentir indesejado por todos.

Vale lembrar que a família precisa conhecer o transtorno e a gravidade da patologia para que a consiga prestar o apoio necessário. Os relatos dos familiares mediante ao profissional contribui para o diagnóstico. A terapia ajudará na compreensão da psicopatologia, e auxiliará o paciente e seus familiares dando a essas umas melhores condições de vida.

\subsection{Transtorno de Humor Bipolar e os seus tipos}

Cardiole et al. (2017) comentam que existem quatro tipos básicos de transtorno bipolar: transtorno bipolar I, transtorno bipolar II, Transtorno Ciclotímico (ciclotimia) e outros transtornos bipolares especificados e não especificados e relacionados, sendo o THB o tipo mais comum. Todos os tipos de transtorno bipolar são caracterizados por episódios de humor extremo, seja pelo excesso, seja pela ausência (Alves, 2016).

O Quadro 1 apresenta os Quatro Tipos De Transtorno Bipolar segundo DSM-5 (2014): 
Quadro 1. Quatro Tipos De Transtorno Bipolar.

\begin{tabular}{|c|l|}
\hline Transtorno Bipolar I & $\begin{array}{l}\text { é uma psicopatologia em que as pessoas experimentam um ou mais episódios de mania. A maioria } \\
\text { das pessoas com diagnóstico de bipolar I terá episódios tanto de mania quanto de depressão, } \\
\text { embora um episódio de depressão não seja necessário para o diagnóstico. Para ser diagnosticado } \\
\text { com bipolar I, os episódios maníacos de uma pessoa devem durar pelo menos sete dias ou ser tão } \\
\text { graves que a hospitalização é necessária. }\end{array}$ \\
\hline Transtorno Bipolar II & $\begin{array}{l}\text { é um subconjunto do transtorno bipolar no qual as pessoas experimentam episódios depressivos } \\
\text { alternando entre episódios hipomaníacos, mas nunca um episódio maníaco "completo". }\end{array}$ \\
\hline $\begin{array}{c}\text { Transtorno Ciclotímico } \\
\text { ou Ciclotimia }\end{array}$ & $\begin{array}{l}\text { é um estado de humor cronicamente instável no qual as pessoas experimentam hipomania e } \\
\text { depressão leve por pelo menos dois anos. Pessoas com ciclotimia podem ter breves períodos de } \\
\text { humor normal, mas esses períodos duram menos de oito semanas. }\end{array}$ \\
\hline $\begin{array}{c}\text { Transtorno Bipolar, } \\
\text { "outro especificado"e } \\
\text { "não especificado" }\end{array}$ & $\begin{array}{l}\text { é quando uma pessoa não atende aos critérios para bipolar I, II ou ciclotimia, mas ainda } \\
\text { experimentou períodos de elevação anormal do humor clinicamente significativa. }\end{array}$ \\
\hline
\end{tabular}

Fonte: DSM-5 (2014).

A principal diferença entre transtornos bipolares e outros transtornos mentais, está na gravidade dos episódios maníacos causados por cada tipo. Scussel et al. (2016) afirmam que uma pessoa com bipolar 1 experimentará um episódio maníaco completo, enquanto uma pessoa com bipolar 2 terá apenas um episódio hipomaníaco (um período que é menos grave do que um episódio maníaco completo).

Amaral (2017) e Da Costa et al. (2017) elucidam que uma pessoa com THB bipolar 1 pode ou não experimentar um episódio depressivo maior, enquanto uma pessoa com outro tipo de bipolaridade, experimentará um episódio depressivo maior.

Os sintomas de um episódio maníaco podem ser graves a ponto de a pessoa necessitar de cuidados hospitalares, de forma que fique impossibilitada de exercer seu trabalho ou qualquer atividade laboral (PetkeviciuS et al., 2020).

Já na visão de Lara (2017), os sujeitos afetados pelo tipo maníaco apresentam episódios caracterizados por exaltação excessiva, irritabilidade, excesso de conversa, fuga de ideias, fala e atividade motora aceleradas. Durante este episódio o indivíduo tem prejuízos significativos na sua vida pessoal, social e profissional e em casos mais graves podem necessitar ser hospitalizados para que não cause danos em si mesmo e para os outros.

\subsection{O sofrimento do indivíduo com Transtorno de Humor Bipolar frente ao preconceito e à discriminação}

Sentir-se estigmatizado é comum entre aqueles que vivem com transtorno mentais. Em todo o mundo, muitas pessoas com psicopatologias são discriminadas, têm oportunidades de trabalho restritas, sentem-se estigmatizadas no trabalho, e têm até mesmo negados os direitos básicos concedidos a outros membros da sociedade. Verifica-se que a maioria do público, desde crianças e adolescentes, a profissionais de saúde mental, e inclusive familiares, mantenha atitudes estigmatizantes em relação às pessoas com transtorno mentais (Silva et al, 2020).

O indivíduo com THB possui uma variação de humor que se alterna em depressivo e maníaco, podendo durar semanas ou meses. A complexidade do THB leva muitas pessoas a desconhecer a psicopatologias o que os leva a enfrentar diferentes estigmas na sociedade, fazendo com que os pacientes a optarem por afastar-se de pessoas, afetando sua qualidade de vida (Kapczinski; Quevedo, 2016).

Nesse sentido, Lara (2017) relatou que as pessoas com THB tendem a fazer suposições gerais sobre minha vida com base em muitos estereótipos falsos que cercam o transtorno bipolar. A sociedade acredita que não podemos trabalhar, não podem ter relacionamentos estáveis, entre outras coisas. Afetam o quadro clínico desse paciente.

Na concepção de Regne et al. (2020), um dos entraves que contribuem para os cenários de sofrimento com THB, são a desinformação e o estigma, que afetam tanto a família, quanto o próprio indivíduo portador de THB, que tem seu tratamento comprometido. 
Para Pereira e Gomes (2017), o estigma público arruína a autoestima dos indivíduos com qualquer tipo de transtorno mental, e por sua causa, diversos não procuram empregos ou lutar por apoio apropriado, ademais, os que encontram acompanhamento abandonam especialistas por apreensão do diagnóstico e da discriminação que ele e a família virão a sofrer.

Magalhães (2021) sinaliza que uma vez em que transtornos mentais são retratados de maneira diferente de questões físicas, a exclusão social torna-se um comum e a sociedade passa a utilizar os transtornos como um padrão de não aceitação para cenários sociais e profissionais.

Ainda sobre o sofrimento de quem sofre com THB, Regne et al. (2020) diz que o paciente "carrega consigo um estigma social, e o ato de revelar a sua existência, seja em si mesmo ou em um familiar, gera medo, o que faz com que muitos prefiram esconder ou negar a psicopatologia". Assim, com a falta de aceitação e receios de exclusão, muitos indivíduos com THB isolam-se do convívio social.

Sobre práticas relacionadas à estigma, Gomes (2018) reproduz que: o estigma no nível social é quando uma parcela da sociedade, um grupo social, endossa estereótipos sobre um grupo estigmatizado - e ocorre como resultado de estereótipos negativos, preconceitos e discriminação em relação aos pacientes psiquiátricos. Já o estigma internalizado, ou autoestigma, é quando os indivíduos acreditam nos rótulos associados à sua condição e endossam os estereótipos como algo relevante para a autoacreditação e autovalorização. O estigma internalizado consiste no descrédito do indivíduo relacionado à sua própria condição, o que se caracteriza como aceitação e introjeção do preconceito alheio. Esse tipo de estigma resulta de um alto nível de insegurança (apud Corrigan et al., 2005).

Deste modo, é notório que as características que a sociedade impõe à personalidade de cada indivíduo têm diversos desdobramentos moldando a personalidade do indivíduo e/ou gerando sofrimento psíquico. As pessoas com THB são rechaçadas pela sociedade que teme à mudança repentina de humor provocadas pelo transtorno e as características de cunho comportamental.

Para Santos (2019) enfrentar o estigma implica que os profissionais de saúde estejam dispostos a promover a relação terapêutica considerando as expectativas dos pacientes e sua participação nas decisões, o que reduz a autodepreciação e o sentimento de descrença, e tende a melhorar a adesão ao tratamento e a qualidade de vida

\section{Considerações Finais}

Observou-se na discussão deste estudo que as pessoas que possuem transtornos bipolar, por não apresentarem comportamentos socialmente aceitos, acabam sendo excluídos do convívio social, pois a sociedade busca imitar o que é certo, procurando no indivíduo qualidades que poderão contribuir mediante ao que a sociedade exige, o indivíduo portador do THB também sofre com as discriminações e preconceito, e até mesmo a desvalorização, onde a sociedade o juga como anormal.

Não somente a família, porém o ato de educar as pessoas de modo geral quanto à sensibilização ao paciente com THB mostrou-se de suma importância, uma vez que o tratamento é feito de forma psicológica, ou seja, tais sentimentos como a autoestima e a aceitação social são fundamentais para o avanço do paciente no tratamento, pois estes estigmas são os obstáculos primários que os que sofrem de THB enfrentam diariamente no vínculo social.

A irritabilidade e o nervosismo também são sintomas predominantes durante estes episódios de THB, ou seja, a sua autoestima encontra-se inflada o indivíduo não concorda com as diferenças existentes no seu convívio social e por isso fica mais propenso a irritasse com atitudes que ele julga errôneas. Por isso é importante sensibilizar a situação real do paciente com THB para aqueles que estão relacionados ao seu convívio diário. 
Percebeu-se que mesmo o indivíduo tendo seu o acompanhamento, ou seja, o tratamento adequando o senso comum faz o julgamento na aceitação de que esse indivíduo possa viver em meio ao convívio social, pois a má informação sobre a psicopatologia os faz ter essas discriminações com o portador de transtorno bipolar.

São diversos os trabalhos como o dos autores: Pereira e Gomes (2017); Regne et al. (2020); Silva et al, 2020; Lara (2017); Fassarella (2019) e tratam o tema na comunidade científica, mas poucos buscam abordar questões sobre os preconceitos e discriminações existentes na sociedade e seus impactos no indivíduo com THB. Deste modo, espera-se assim, haver uma reflexão acerca do atual senso comum instaurado na sociedade sobre o THB e seus comportamentos, além da propagação de conhecimentos na sociedade sobre os tratamentos que o paciente enfrenta, a fim de reduzir como um todo este estigma sobre o THB.

\section{Referências}

Almeida, B. R. A., de Almeida, C. G. S., de Oliveira, C. C. M., Machado, D. C. A., Ruckl, S., \& Andrade, V. A. (2018). Atualização no tratamento do transtorno bipolar: o impacto da psicoeducação familiar. Revista Portuguesa de Psiquiatria e Saúde Mental, (3), 11-17.

Alves, R. O. P. (2016). Comorbidade entre o Transtorno Bipolar Tipo I e Transtornos de Personalidade: avaliação das características clínicas e sociodemográficas. Monografia, como exigência parcial e obrigatória para conclusão do Curso de Medicina da Faculdade de Medicina da Bahia (FMB), da Universidade Federal da Bahia (UFBA)

Amaral, C. E. C. D. (2017). Transtorno bipolar: impacto das comorbidades no diagnóstico, tratamento e prognóstico. Curso de Medicina, Universidade Federal do Maranhão, Hospital Universitário Presidente Dutra,

Aragão, J. W. M. D., \& Mendes Neta, M. A. H. (2017). Metodologia científica. Salvador: UFBA, Faculdade de Educação, Superintendência de Educação a Distância, 2017

Barlow, D. H. (2016). Manual clínico dos transtornos psicológicos: tratamento passo a passo. (5a ed.), Artmed Editora, 2016.

Bauer, M. W., \& Gaskell, G. (2017). Pesquisa qualitativa com texto, imagem e som: um manual prático. Petrópolis: Editora Vozes Limitada.

Bell, J. (2016). Projeto de pesquisa: guia para pesquisadores iniciantes em educação, saúde e ciências sociais. Artmed.

Casanova, F. M., Sussulini, A., Madrid, K. C., \& Arruda, M. A. (2018). Avaliação metalômica do soro sanguíneo de pacientes com transtorno bipolar, transtorno esquizoafetivo e esquizofrenia. Revista dos Trabalhos de Iniciação Científica da UNICAMP, (26).

Caponi, S. (2014). O DSM-V como dispositivo de segurança. Physis: Revista de Saúde Coletiva, 24, 741-763.

Cordioli, AV, de Souza Vivan, A., \& Braga, DT (2017). Vencendo o Transtorno Obsessivo-Compulsivo-: Manual de Terapia Cognitivo-Comportamental para Pacientes e Terapeutas. Artmed Editora. 3. ed. Porto Alegre: Artmed.

Da Costa, R. S., dos Santos, D. R., \& Soares, M. R. Z. (2016). Intervenção psicológica em grupo para pacientes com diagnóstico de Transtorno Bipolar: uma revisão da literatura. Contextos Clínicos, 9(2), 225-239.

Da Costa, R. S. (2016). Transtorno Bipolar: Contribuições de uma intervenção analítico--comportamental em grupo (Doctoral dissertation, Dissertação de Mestrado), Universidade Estadual de Londrina, Londrina, PR).

da Costa, R. S., Soares, M. R. Z., \& Grossi, R. (2017). Estrutura das sessões de uma intervenção analítico-comportamental em grupo para pessoas diagnosticadas com transtorno bipolar. Acta Comportamentalia: Revista Latina de Análisis de Comportamiento, 25(1), 57-72.

Dalgalarrondo, P. (2018). Psicopatologia e semiologia dos transtornos mentais. Artmed Editora. Artmed Editora.

Deminco, M. (2019). Transtorno Afetivo Bipolar-Visão Geral. Clube de Autores (managed).

de Oliveira, R. R., Kuhn, D., Rigoli, M. M., \& Bücker, J. (2019). Contribuições e principais intervenções da terapia cognitivo-comportamental no tratamento do transtorno bipolar. Aletheia, 52(2).

de QUEIROZ, J. B., \& da COSTA, R. F. (2017). Transtorno Bipolar: Diagnóstico, Características E Intervenção Psicológica. In: Anais de Psicologia do Unifunec, v. 4, n. 4 .

American Psychiatric Association. (2014). DSM-5: Manual diagnóstico e estatístico de transtornos mentais. Artmed Editora.

Fassarella, M. R., de Oliveira, M. E. P., \& de Almeida, M. Z. T. Um olhar sobre o transtorno bipolar e possíveis dificuldades de adaptação social. Principais transtornos psíquicos na contemporaneidade, 43.

Gomes, D. R. A. S. Estigma internalizado em pacientes com transtorno de humor e fatores associados (Doctoral dissertation, Universidade de São Paulo).

Lara, C. D. A. (2017). O jogo metafórico do transtorno bipolar: presença e ausência na revista Debates em psiquiatria. Dissertação (mestrado) Universidade Federal de Santa Catarina, Centro de Comunicação e Expressão, Programa de Pós-Graduação em Linguistica, Florianópolis. 
Research, Society and Development, v. 10, n. 12, e547101220571, 2021

(CC BY 4.0) | ISSN 2525-3409 | DOI: http://dx.doi.org/10.33448/rsd-v10i12.20571

Kapczinski, F., Vieta, E., Magalhães, P. V., \& Berk, M. (2016). Neuroprogressão e Estadiamento no Transtorno Bipolar. Artmed Editora.

Lima, N. N. R., \& Neto, M. L. R. (2019). O Transtorno Bipolar do Humor em Pacientes Atendidos em Unidade Básica de Saúde/Bipolar Disorder in Primary Care Patients. ID on line Revista de Psicologia, 13(48), 796-806.

Magalhães, T. T. B (2021). Análise das repercussões laborais de portadores de Depressão Unipolar e Bipolar: um estudo transversal. Trabalho de conclusão de curso ao Centro Universitário de Brasília (UniCEUB), como parte da exigência para obtenção do título de bacharel em Medicina.

MARQUES, N. et al. (2018). Transtorno Bipolar. In: V. 3, n. 1 - I Semana de Psicologia-FAGOC.

Moreno, R. A., Moreno, D., Bio, D. S., \& David, D. P. (2015). Aprendendo a Viver com o Transtorno Bipolar: Manual Educativo. Artmed Editora.

Petkevicius, G. A M., Roscoche, K. G. C., Soares, A. B. S., de Sousa, A. A. S., de Aguiar, A. S. C., \& Felício, J. F. (2020). Perfil clínico-epidemiológico de pessoas com transtorno bipolar em internação psiquiátrica. Research, Society and Development, 9(9), e394997282-e394997282.

Pereira, L. F., \& Gomes, K. M. (2017). O olhar do paciente do CAPSII sobre a psicofobia. Revista de Extensão, 2(1), 128-140.

Regne, G. R. S., dos Santos Reinaldo, A. M., da Silveira, B. V., \& Gomes, N. D. M. R (2020). Transtornos do humor, sintomas e tratamento na perspectiva dos familiares. SMAD, Rev. Eletrônica Saúde Mental Álcool Drogas.

Santos, K. G. D. (2019). Repercussões do transtorno bipolar na família e suas implicações para a enfermagem: uma revisão integrativa. Trabalho de Conclusão de Curso (Bacharel em Enfermagem) -Universidade Federal do Rio Grande do Sul.

Schiavo, L., \& Nunes, C. P. (2019). Tratamento do transtorno bipolar: indagação de uma nova abordagem. Revista de Medicina de Família e Saúde Mental, $1(1)$.

Silva, R. D. A. D., Tancini, M. B., Cheniaux, E., \& Mograbi, D. C. (2020). Metacognição no transtorno bipolar: uma revisão sistemática. Jornal Brasileiro de Psiquiatria, 69, 131-139.

Scussel, F., Salvador, L. C., Brandão, L. S., \& Feier, G. (2016). Perfil clínico dos pacientes com transtorno bipolar atendidos em um ambulatório especializado na região sul catarinense. Arquivos Catarinenses de Medicina, 45(4), 3-10.

Whitbourne, S. K., \& Halgin, R. P. (2015). Psicopatologia: perspectivas clínicas dos transtornos psicológicos. AMGH. 